\title{
A modified calculation of particle buoyant forces in vibro- fluidized beds
}

\author{
Zhixiong Zhang ${ }^{1,2}$, Xihua $\mathrm{Chu}^{1 *}$, Yanran Wang ${ }^{1}$ \\ ${ }^{1}$ School of Civil Engineering, Wuhan University, Wuhan 430072, China \\ ${ }^{2}$ CITIC general institute of architectural design and research Co., Ltd., Wuhan 430014, China
}

\begin{abstract}
Segregation of granular materials under vibration or flow conditions such as the Brazil nut effect has been well known, however, there is yet no consensus mechanisms to explain this phenomenon. This study attempts to investigate particle buoyant forces in the segregation process. To explain the difference of the segregation behavior for the large particle with different size, a modified calculation method of particle buoyant force is suggested for considering the effect of particle size ratio. A simple verification illustrates its validity.
\end{abstract}

\section{Introduction}

Granular materials attempt to exhibit spatially segregation of different components after vibration, such as the Brazil nut effect, which is self-organization displayed by systems that are out of equilibrium. To explain this segregation behavior, several mechanisms have been proposed, such as void-filling [1], convection [2], condensation [3], and some new mechanisms, such as buoyancy [4], asymmetric flux model [5], material point method [6], etc. Void-filling mechanism and convection mechanism are based on experimental observation, which can explain Brazil nut effect intuitively, but isn't suitable for reverse Brazil nut effect, and is also limited to the boundary conditions [7]. The condensation mechanism can eliminate the influence of boundary and explain the reverse Brazil nut effect, but it ignores some basic physical properties of particles such as friction, collision, etc. There is yet no accepted approach to explain such segregation behavior of particle materials.

This study is based on buoyancy mechanism, to explain the difference of the floating behavior when the large particle size changes, we suggested a modified method for calculating the buoyancy of large particles based on the size ratio. In this study, the numerical investigations are based on the discrete element method proposed by Cundall and Strack [8], linear contact is used to calculate the force between particles.

\section{Buoyancy mechanism in granular flow}

In 1986, Campbell [9] has defined the stress tensor in a two-dimensional granular shear flow which is composed of streaming stress tensor and collisional stress tensor. The streaming stress tensor is

$$
\begin{gathered}
\tau_{s}^{*}=\rho_{p} v\left(\begin{array}{cc}
\left\langle u^{\prime 2}\right\rangle & \left\langle u^{\prime} v^{\prime}\right\rangle \\
\left\langle u^{\prime} v^{\prime}\right\rangle & \left\langle v^{\prime 2}\right\rangle
\end{array}\right) \\
p^{\prime} q^{\prime}=\langle p q\rangle-\langle p\rangle\langle q\rangle
\end{gathered}
$$

where $\tau_{s}^{*}$ is the streaming stress tensor, $\rho_{p}$ is particle density, $v$ is the particle volume fraction, $u$ and $v$ is particle velocity in $x$ and $y$ directions respectively, \langle\rangle represents calculate average value. The collisional stress tensor is

$$
\begin{gathered}
\boldsymbol{J}=m\left(\frac{1}{2}(1+\varepsilon)(\boldsymbol{q} \cdot \boldsymbol{k}) \cdot \boldsymbol{k}+\frac{1}{2(1+1 / \beta)}(\boldsymbol{q}-\boldsymbol{q} \cdot \boldsymbol{k}) \cdot \boldsymbol{k}+R\left(\boldsymbol{\omega}_{1}+\boldsymbol{\omega}_{1}\right) \times \boldsymbol{k}\right) \\
\boldsymbol{\tau}_{c}=2 R[\boldsymbol{J} \boldsymbol{k}]
\end{gathered}
$$

where $\boldsymbol{q}=\boldsymbol{u}_{1}-\boldsymbol{u}_{2}, \boldsymbol{u}_{1}, \boldsymbol{u}_{2}$ is particle 1 and 2 velocity before collision, $\omega_{1}, \omega_{2}$ is rotation rates, $\boldsymbol{k}=\left(\boldsymbol{x}_{1}-\boldsymbol{x}_{2}\right) /\left\|\boldsymbol{x}_{1}-\boldsymbol{x}_{2}\right\|, \quad \beta$ is the square of the ratio of the particle radius of gyration to the particle radius, $m$ is particle mass, $R$ is particle radius, $\mathcal{E}$ is coefficient of restitution, [ ] represents calculate average value among statistical area and time.

In 2001, Shishodia [4] has defined the pressure in the vibro-fluidized particle bed based on the stress tensor proposed by Campbell, the particle pressure is

$$
P=\frac{1}{2}\left(\tau_{s, x x}+\tau_{s, y y}+\tau_{c, x x}+\tau_{c, y y}\right)
$$

where $P$ is the pressure, $\tau_{s, x x}, \tau_{s, y y}$ is the streaming stress tensor in $x$ and $y$ directions respectively, $\tau_{c, x x}, \tau_{c, y y}$ is the collisional stress tensor in $x$ and $y$ directions respectively. Under two-dimensional condition, the particle buoyancy is

\footnotetext{
* Corresponding author: chuxh@whu.edu.cn

A video is available at https://doi.org/10.48448/jx7d-pr40
} 


$$
F_{p}=\frac{\pi}{4} d^{2} \frac{d p}{d y}
$$

where $F_{p}$ is particle buoyancy, $d$ is radius of large particle, $d p / d y$ is local pressure gradient.

\section{Problems in the application of buoyancy mechanism}

Shishodia's simulation model and the floating process of large particles are shown in Figure 1. Sinusoidal displacement load is applied to the bottom wall. The large particles rise first and then stabilize at an equilibrium position, where particle buoyancy and gravity balance. (a)

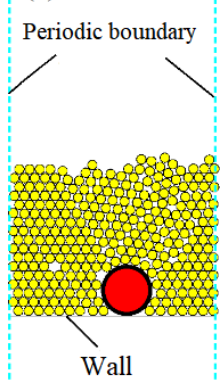

(b)

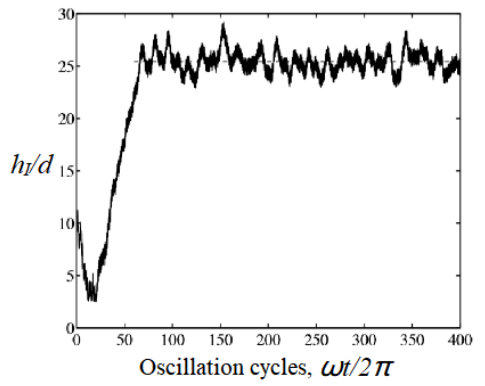

Fig. 1. (a) Shishodia's simulation model; (b) A plot of the instantaneous height of the impurity (Shishodia, 2001)

In 2005, Huerta [10] has designed a device to measure the buoyancy of large particles in the vibrating bed, shown in Figure 2. It can be seen that the buoyancy of large particles is directly proportional to its volume.

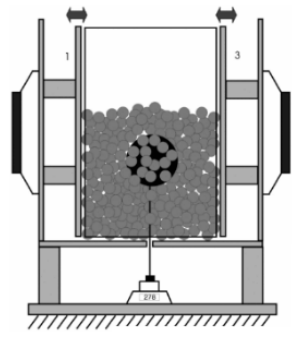

(a)

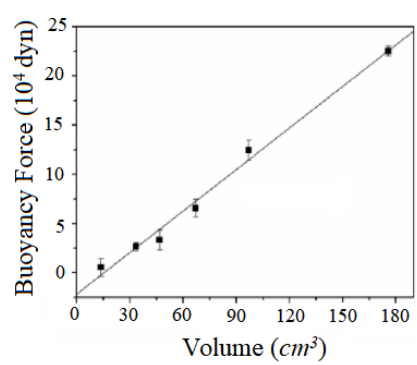

(b)
Fig. 2. (a) Particle buoyancy mesure device; (b) The relationship between particle buoyancy and its volume (Huerta, 2005)

The buoyancy mechanism has been confirmed in both numerical simulation and physical experiment. However, the effects of particle size ratio were neglected in Shishodia's numerical work. In this work, we conducted a series of numerical experiments based on the discrete element method to investigate buoyance forces for samples with different particle size ratio. The simulation model parameters are shown in table 1 , and the results are shown in Figure 3.

The numerical results show two obvious facts: first, the large particle won't rise when size ratio is small enough; second, larger particles rise faster than smaller particles. The above phenomena have been discussed in relevant literatures $[7,11,12]$. However, according to the buoyancy calculation method proposed by Shishodia, the equilibrium position of large particles is only related to their density, the influence of particle size ratio is out of consideration.

Table 1. Simulation parameters

\begin{tabular}{|l|l|}
\hline Parameters & Value \\
\hline $\begin{array}{l}\text { Vibration device size (width, height) } \\
(\mathrm{m})\end{array}$ & $0.2,0.6$ \\
\hline $\begin{array}{l}\text { Particle number (large particle, small } \\
\text { particle) }\end{array}$ & 1,300 \\
\hline Diameter of small particle $(\mathrm{m})$ & 0.01 \\
\hline $\begin{array}{l}\text { Particle dengsity (large particle, small } \\
\text { particle) }(\mathrm{kg} / \mathrm{m} 3)\end{array}$ & 750,2500 \\
\hline $\begin{array}{l}\text { Friction coefficient, normal critical } \\
\text { damping ratio }\end{array}$ & $0,0.16$ \\
\hline $\begin{array}{l}\text { Elasticity modulus of wall (normal } \\
\text { and tangential) }(\mathrm{N} / \mathrm{m})\end{array}$ & $2 \times 10^{11}$ \\
\hline $\begin{array}{l}\text { Elasticity modulus of particle (normal } \\
\text { and tangential })(\mathrm{N} / \mathrm{m})\end{array}$ & $1 \times 10^{7}$ \\
\hline Particle local damping coefficient & 0.056 \\
\hline Time step $(\mathrm{s})$ & $1 \times 10^{-6}$ \\
\hline Load (amplitude $(\mathrm{m})$, frequency $)$ & $0.0068,29.85$ \\
\hline
\end{tabular}

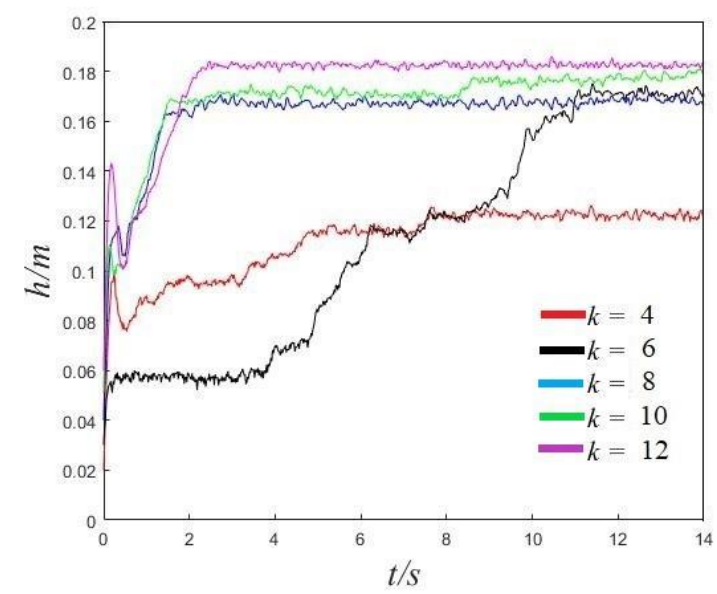

Fig. 3. The large particle's upward movement under different size ratio ( $k$ is the size ratio)

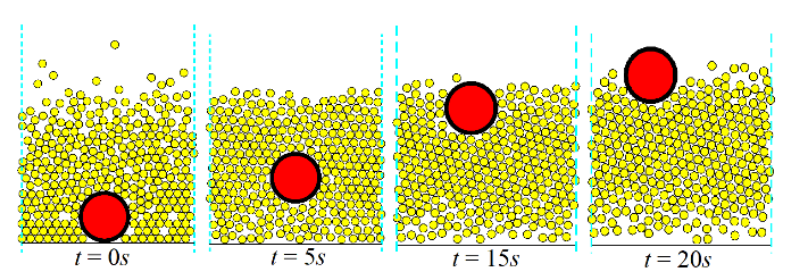

Fig. 4. The rising process of large particle $(k=6)$

\section{A modified calculation of particle buoyant forces}

According to the buoyancy calculation method proposed by Shishodia, the pressure in the particle bed is similar to the liquid pressure, which is continuous and of equal magnitude in all directions. However, the movement of discrete particles is based on the collision and contact between particles, which is discrete, and the discontinuity will be more obvious with the decrease of particle size ratio. 
The large particles rise approximately at a constant speed based on Figure 3, the particle gravity resistance and buoyancy are balanced during this process.

$$
\boldsymbol{F}_{p, r}+\boldsymbol{F}_{d, r}+\boldsymbol{G}=0
$$

$\boldsymbol{F}_{p, r}$ is the real particle buoyancy, $\boldsymbol{F}_{d, r}$ is the real particle resistance, $\boldsymbol{G}$ is the particle gravity.

Considering the influence of particle size ratio, the original resistance and original buoyancy are multiplied by the same reduction coefficient $\alpha$. Here, the buoyancy and resistance of large particles are both based on the interaction between large and small particles, so the influence of the particle size ratio factor should be taken into account at the same time.

$$
\alpha \boldsymbol{F}_{p}+\alpha \boldsymbol{F}_{d}+\boldsymbol{G}=0
$$

$\boldsymbol{F}_{p}$ is the original particle buoyancy, $\boldsymbol{F}_{d}$ is the original particle resistance.

In 2003, Chehata [13] has designed experiments to measure the drag force of the cylinder in the dense particle flow system, which is

$$
F_{D}=\frac{1}{2} C_{D} \rho v_{\max } U_{\infty}^{2}(D+d)(L-d)
$$

where $\rho$ is density of small particle, $v_{\max }$ is the maximum volume fraction of small particles near the cylinder, $U_{\infty}$ is the velocity of small particles near the cylinder, $D$ is the diameter of cylinder, $L$ is the height of cylinder, $d$ is the small particle diameter, $C_{D}$ is the drag coefficient.

The relationship between drag coefficient with Froude number is shown in Figure 5.

$$
F_{r}=\frac{U_{\infty}}{\sqrt{g(D+d)}}
$$

$F_{r}$ is particle Froude number, $g$ is acceleration of gravity.
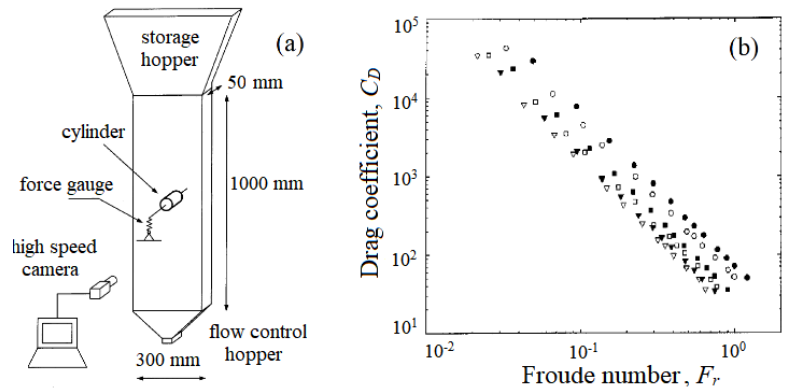

Fig. 5. (a)A schematic of the experimental apparatus; (b)The relationship between drag coefficient with Froude number (Chehata, 2003)

Acording to Figure 5, the relationship between drag coefficient with Froude number is

$$
\log _{10} C_{D}=-1.882 \log _{10} F_{r}+\lambda
$$

We use Chehata's method for calculating the drag force of large particles and the relationship between the drag coefficient and the Froude number for reference. It should be noted that there is friction between particles in the experimental state, while the numerical model established in this study does not consider it. As Huerta's experiment results shown, when $k$ is large enough, the buoyancy of large particles is directly proportional to its volume, which means $\alpha$ should be close to 1 . We have tested that only if $\lambda$ is assigned to 0.18 , the above relationship of $k$ and $\alpha$ could be established.

Based on the data from Figure 3, first we use Eq.10, Eq11, Eq.9 and Eq.6 to calculate the particle original resistance and original buoyancy, then we insert the resistance and the buoyancy in Eq.8 to get the value of $\alpha$, the relationship of $k$ and $\alpha$ is shown in Figure 6 .

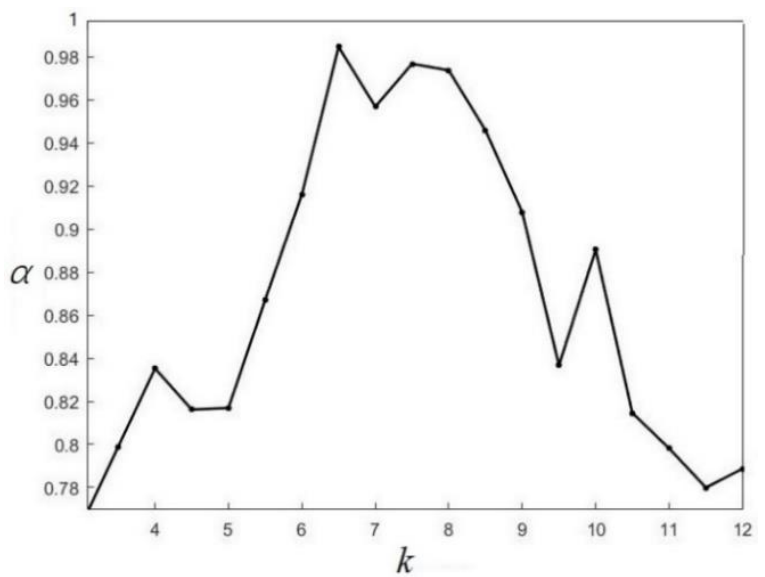

Fig. 6. The relationship between reduction coefficient with size ratio

When $k \geq 7.77$, the curve shows a downward trend, which isn't consistent with the ideal situation. Because we didn't consider the large particle' influence on pressure distribution. There are some facts in Huerta' experiments to support our discoveries in this study: first, the particle size and buoyancy accord with a direct proportion relationship when the size ratio is very large; second, the line does not pass through the origin in Figure 2, which means direct proportion relationship may not establish under small size ratio.

The relationship between the buoyancy of a large particle and its volume should be like in Figure 7, when the particle size is small the slope of the curve has a process from small to large.

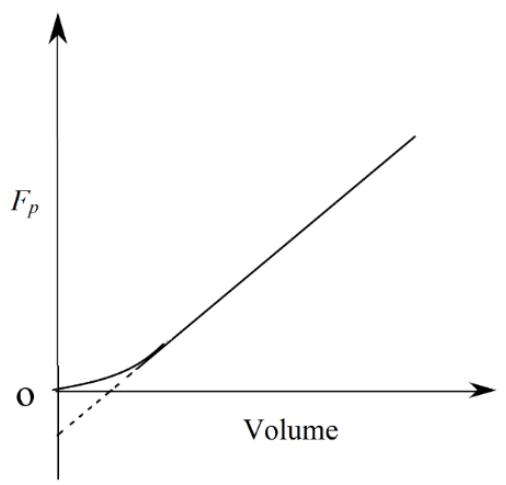

Fig. 7. The real relationship of particle buoyancy with its volume

Then, we suggested that the relationship between the reduction coefficient and particle size ratio is

$$
\alpha= \begin{cases}0.0504 k+0.6083 & , 3.5 \leq k<7.77 \\ 1 & , 7.77 \leq k\end{cases}
$$


When $1<k<3.5$, large particles don't raise so we didn't consider it. To verify the validity of Eq.12, we added simulations when the particle size ratio was 3.8, $4.3,4.8,5.3,5.8,6.3,6.8$ and the comparison of reduction coefficient between predicted and actual are shown in table 2 .

Table 2. Comparing the predicted value with the actual value

\begin{tabular}{|c|c|c|c|c|}
\hline$k$ & $v(m / s)$ & $\alpha_{p}$ & $\alpha_{r}$ & $s_{\alpha}=\left|\alpha_{p}-\alpha_{r}\right| / \alpha_{r}$ \\
\hline 3.8 & 0.0038 & 0.7998 & 0.7433 & $7.59 \%$ \\
\hline 4.3 & 0.0116 & 0.8250 & 0.8594 & $4.00 \%$ \\
\hline 4.8 & 0.0124 & 0.8502 & 0.7964 & $6.74 \%$ \\
\hline 5.3 & 0.0212 & 0.8754 & 0.8391 & $4.32 \%$ \\
\hline 5.8 & 0.0397 & 0.9006 & 0.9214 & $2.25 \%$ \\
\hline 6.3 & 0.0315 & 0.9258 & 0.8164 & $13.39 \%$ \\
\hline 6.8 & 0.0518 & 0.9510 & 0.8784 & $8.26 \%$ \\
\hline
\end{tabular}

It can be seen from table 2 that the difference between the actual value and the predicted value of the reduction coefficient is vary within $15 \%$, indicating that Eq.12 is valid.

\section{Conclusions}

In this paper, particle vibration segregation behavior was studied based on the buoyancy mechanism proposed by Shishodia. It was found that the existing buoyancy calculation method could not explain the difference of the floating behavior of large particles when the particle size ratio changed, so a modified calculation of particle buoyant forces was suggested. And the conclusions were as follows:

When the size of the large particle and small particle is close, the large particle does not rise, so we didn't consider it; when $3.5 \leqslant k<7.77$, the predicted buoyancy of large particles should be multiplied by the reduction coefficient, and the smaller the particle size ratio, the more degree of reducing the buoyancy; when $k \geqslant 7.77$, the reduction coefficient is close to 1 , the effect of particle size ratio can be neglected.

\section{Acknowledgment}

Funding: this work was supported by the National Nature Science Foundation of China [grant numbers: 11772237, 11472196]

\section{References}

[1] J.C. Williams, Fuel Soc. J., 14, 29, (1963)

[2] J.B. Knight, et al., Phys. Rev. Lett. ,70, 3728, (1993)

[3] D.C. Hong, et al., Phys. Rev. Lett., 86, 3423, (2001)

[4] N. Shishodia and C.R. Wassgren, Phys. Rev. Lett., 87, 084302, (2001)

[5] P. Gajjar and J.M.N.T. Gray, J. Fluid Mech., $757,297,(1993)$

[6] M.L. Fei and Q.C Sun, Simulation of size segregation in multi-regimes granular flow with material point method, in CCTAM-2019, CCTAM, 25-28 August 2019, HangZhou, China (2019)

[7] K. Liffman, et al., Granul Matter 3, 205, (2001)

[8] P.A. Cundall and O.D.L. Strack, Geotechnique, 29 , 47, (1979)

[9] C.S. Campbell and A. Gong, J. Fluid Mech., 164, 107, (1986)

[10] D.A. Huerta, et al., Phys. Rev. E, 72, 031307, (2005)

[11] A.D. Rosato, et al., Powder Technol., 66, 149, (1991)

[12] K. Ahmad, and I.J. Smalley, Powder Technol., 8, 69, (1973)

[13] D. Chehata, et al., Phys. Fluids, 15, 1622, (2003) 